\title{
Ensilado de maíz en dietas para postlarva de camarón de agua dulce Macrobrachium rosenbergii*
}

\author{
Maurício G. Coelho-Emerenciano ${ }^{1}$ \& Wilson Massamitu-Furuya ${ }^{1}$ \\ ${ }^{1}$ Departamento de Zootecnia, Universidad Estatal de Maringá, Av. Colombo 5.790, zona 7 \\ Maringá-PR, Brasil
}

\begin{abstract}
RESUMEN. Se evalúa la substitución del maíz por ensilado de granos de maíz en dietas para camarones de agua dulce Macrobrachium rosenbergii. Se utilizaron 640 postlarvas de camarones $(0,12 \pm 0,04 \mathrm{~g} ; 24,65 \pm 2,53 \mathrm{~mm})$, que fueron distribuidas aleatoriamente en cuatro tratamientos y cuatro réplicas. En cada unidad experimental constituida por una jaula de $130 \mathrm{~L}$ de capacidad, se introdujeron 40 postlarvas. Se utilizó una dieta referencia con 37,7\% de proteína bruta, y $4.343 \mathrm{Kcal}$ de energía bruta $\mathrm{kg}^{-1}$. El ensilado de maíz sustituyó el maíz de la dieta de referencia en concentraciones de $0,8,16$ y $24 \%$. No se observaron los efectos de los tratamientos sobre las variables del consumo de ración, factor de conversión de la dieta, eficiencia proteica y supervivencia. La ganancia de peso y la biomasa final crecieron linealmente con respecto a la inclusión de ensilado de maíz en las dietas y se ajustaron a un modelo de regresión lineal. Se concluyó que el maíz puede ser totalmente sustituido por ensilado de maíz en las dietas para postlarvas de camarones de agua dulce M. rosenbergii, que tienen hasta un $24 \%$ de ese ingrediente.
\end{abstract}

Palabras clave: Macrobrachium rosenbergii, camarones de agua dulce, ensilado de maíz, postlarvas, alimento, Brasil.

\section{Corn silage in the diet of postlarval freshwater prawns Macrobrachium rosenbergii*}

\begin{abstract}
The present work evaluates the substitution of corn silage for corn in the diets of the freshwater prawns Macrobrachium rosenbergii. The 640 postlarvae $(0.12 \pm 0.04 \mathrm{~g} ; 24.65 \pm 2.53 \mathrm{~mm})$ used in this study were distributed randomly in four treatments and four replicates. Each experimental unit consisted of a cage ( $130 \mathrm{~L}$ volume) and 40 postlarvae. The reference diet was $37.7 \%$ gross protein and $4.343 \mathrm{Kcal}$ brute energy $\cdot \mathrm{kg}^{-1}$. Concentrations of $0,8,16$, and $24 \%$ corn silage substituted the corn used in the reference diet. The treatments were not observed to affect the following variables: feed consumption, feed conversion ratio, protein efficiency, and survival. Weight gain and final biomass grew lineally with regard to the inclusion of corn silage in the diets; both were fit to a lineal regression model. In conclusion, corn silage (up to $24 \%$ ) can completely substitute corn in the diets of the post-larval freshwater prawns Macrobrachium rosenbergii.
\end{abstract}

Key words: Macrobrachium rosenbergii, freshwater prawns, corn silage, postlarvae, food, Brazil.

Autor corresponsal: Maurício G. Coelho Emerenciano (mauricioemerenciano@hotmail.com).

\section{INTRODUCCIÓN}

El cultivo de camarones de agua dulce es una de las actividades de la acuicultura que más crece en Brasil. Se estima que en el año 2000, la producción fue mayor de 200.000 ton, valorizada en más de
U\$ 1 millón. Esto corresponde al 20\% del volumen total producido por el sector de camarones marinos, porcentaje que históricamente fue de alrededor de $5 \%$ (New, 2000).

El camarón de agua dulce Macrobrachium rosenbergii es un crustáceo de origen Indo-Pacífico,

* Trabajo presentado en el XXV Congreso de Ciencias del Mar de Chile y XI Congreso Latinoamericano de Ciencias del Mar (COLACMAR), realizados en Viña del Mar, entre el 16 y 20 de mayo de 2005. 
ampliamente distribuido en regiones tropicales y subtropicales de varios países asiáticos. Esta especie se encuentra durante todo el año pudiendo hallarse tanto en aguas dulces como salobres, vive en la mayor parte de los ríos y se encuentra hasta $200 \mathrm{~km}$ de la costa. Es una especie omnívora, con régimen de alimentación constante y su dieta incluye gusanos e insectos acuáticos, pequeños moluscos, peces, crustáceos, semillas, frutas, algas, hojas y tallos blandos de plantas acuáticas (Ling, 1969).

La producción de camarones de agua dulce está asociada directamente a la producción de postlarvas que en la actualidad es ampliamente conocida (Valenti, 1998). Entretanto, la evaluación de fuentes alternativas de proteína y energía que puedan ser utilizadas en el cultivo intensivo de crustáceos es importante para mejorar el retorno económico (Millamena \& Triño, 1997), principalmente cuando es sometido a algún tipo de procesamiento mejor a la utilización del almidón (Bombeo-Tuburan et al., 1995; Davis \& Arnold, 1995; Cruz-Suarez et al., 2001).

Las fuentes proteicas de origen vegetal se evaluaron, recientemente, en raciones para camarones por Sudaryono et al. (1999) y Bautista-Teruel et al. (2003), los cuales encontraron que los camarones utilizan con bastante eficiencia las variadas fuentes de proteína de origen vegetal, pero todavía falta información relacionada con el valor nutricional de fuentes energéticas de origen vegetal, que están presentes en valores de 15 a $30 \%$ como ingredientes de las dietas.

En Brasil ha aumentado el uso del ensilado de grano húmedo en raciones para animales. El ensilado permite un aumento en la digestibilidad del almidón de los granos, por las modificaciones causadas en el almidón durante el procesamiento de la materia prima (Jobim et al., 2001). Por ello son importantes los estudios que puedan evaluar la utilización del ensilado de maíz en raciones para camarones de Malasia, considerando los posibles beneficios del ensilado para incrementar su palatabilidad y valor nutritivo.

Las dietas atrayentes y de alta digestibilidad son deseables para que los camarones puedan obtener un buen crecimiento y supervivencia en cultivos intensivos. El maíz es el cereal más utilizado como fuente energética en raciones utilizadas para alimentar camarones en Brasil y su sustitución por el ensilado de maíz puede contribuir a un mayor consumo de la dieta, porque el proceso de ensilado puede mejorar la palatabilidad del maíz así como su valor nutritivo.
Debido a que este proceso aumenta la digestibilidad del almidón y consecuentemente, la disponibilidad de nutrientes y el valor energético de la dieta, para obtener un adecuado desempeño con mínimos daños al medio ambiente.

El presente trabajo tiene como objetivo evaluar el efecto de la sustitución del maíz por el ensilado de maíz en raciones para camarones de agua dulce $M$. rosenbergii, entre 60 y 105 días de edad.

\section{MATERIALES Y MÉTODOS}

El estudio fue realizado en el Laboratorio de Acuicultura de la Universidad Estatal de Maringá, de febrero a marzo de 2004. Se utilizaron 640 postlarvas de camarones $(0,12 \pm 0,04 \mathrm{~g} ; 24,65 \pm 2,53 \mathrm{~mm})$, adquiridas en el Centro de Acuicultura de la Universidad Estatal Paulista (CAUNESP), Jaboticabal-SP. Las larvas se distribuyeron en cuatro jaulas de $130 \mathrm{~L}$ de capacidad, cada una de ellas con distintas dietas. Las jaulas se mantuvieron en un estanque de fibro-cemento de 800 Ly se efectuaron cuatro réplicas de este experimento. La aireación fue mantenida en forma constante por rocas porosas acopladas a un aireador central. La tasa de renovación diaria del agua en las jaulas fue de $20 \%$ y el sistema de filtración estaba constituido por un biofiltro, que tenía como substrato fragmentos de conchas de moluscos marinos.

La dieta de referencia utilizada contenía 37,7\% de proteína bruta y $4.343 \mathrm{kcal}$ de energía bruta $\cdot \mathrm{kg}^{-1}$. Se evaluaron dietas con 0, 8, 16 y 24\% de adición del ensilado de maíz. Este ensilado substituyó el maíz de la dieta referencia, siendo distribuido dos veces al día, sin restricciones de acuerdo con el consumo.

Los ingredientes (maíz, ensilado de maíz, harina de soya y harina de peces) fueron molidos (diámetro menor o igual a $0,35 \mathrm{~mm}$ ) y peletizados en una prensa manual. Para la peletización, se agregó agua en la proporción de $15 \%$ del peso seco de la ración y luego, se deshidrató en estufa de ventilación forzada $\left(50^{\circ} \mathrm{C}\right)$ durante $18 \mathrm{~h}$. Los gránulos fueron desintegrados $\mathrm{y}$ seleccionados en un colador de metal (Tyler 28).

Todos los camarones fueron pesados y medidos al inicio y final del experimento. Se calculó la ganancia en peso (peso medio final-peso medio inicial), factor de conversión de la dieta (consumo de la ración/aumento en peso), eficiencia proteica (aumento en peso/proteína consumida), porcentaje de supervivencia (número de animales vivos al final del experimento/total de animales $\mathrm{x} 100$ ) y biomasa final en cada unidad experimental. 
La temperatura fue registrada dos veces al día; mientras que el $\mathrm{pH}$, la conductividad eléctrica y el oxígeno disuelto fueron controlados cada siete días con ayuda de un "kit" digital portátil. Los datos fueron sometidos al análisis de variación y regresión polinomial, mediante el programa Sistemas de Análisis Estadísticas y Genéticas (Euclydes, 1983).

\section{RESULTADOS}

Durante las experiencias, la temperatura, $\mathrm{pH}$, conductividad eléctrica y oxígeno disuelto se mantuvieron estables y no alcanzaron los niveles letales para esta especie (Valenti, 1998). No se observaron efectos de los tratamientos sobre las variables: consumo de ración, factor de conversión de la dieta, eficiencia proteica y supervivencia $(p>0,05)$. El aumento en peso y la biomasa final aumentaron linealmente ( $\mathrm{p}$ $<0,05)$, a medida que aumentó la concentración del ensilado de maíz. La mayor disponibilidad del almidón, produjo un mayor aprovechamiento de la ración por animal y consecuentemente, un mayor crecimiento y supervivencia.

Con el uso del test de Dunnett se observó que el factor de conversión de la dieta y la eficiencia proteica obtenida en los camarones alimentados con un $24 \%$ de substitución de ensilado de maíz fueron mejores $(p<0,05)$, si se compara con los valores obtenidos en los demás tratamientos.

\section{DISCUSIÓN}

Dietas atractivas y de alta digestibilidad son de importancia para que los camarones tengan un adecuado crecimiento y supervivencia. El maíz es el ingrediente más utilizado como fuente de enegía en dietas para camarones de agua dulce. Su sustitución por ensilado de maíz puede mejorar la posibilidad de las mismas, así como su valor nutritivo, ya que el ensilado promueve una mejor digestibilidad del almidón y por lo tanto, un mejor aprovechamiento de la energía y algunos nutrientes de la dieta.

Los resultados obtenidos en la presente investigación están de acuerdo con los resultados obtenidos en juveniles de abalones (Viana et al., 1994). Se estima que los resultados obtenidos se deben a una mayor palatabilidad. Según Valenti (1998), el almidón promueve un mayor crecimiento y supervivencia en los crustáceos, en comparación con la glucosa. En cuanto a los lípidos, la necesidad de estos es menos evidente.
Tabla 1. Composición porcentual de la ración de referencia. 1) Suplemento mineral y vitamínico (Supremas), composición por kilo de producto: vit. A: 1.200 .000 UI, vit. $D_{3}: 200.000$ UI, vit. E: $12.000 \mathrm{mg}$, vit. $K_{3}: 2.400 \mathrm{mg}$, vit. $B_{1}: 4.800 \mathrm{mg}$, vit. $B_{2}: 4.800 \mathrm{mg}$, vit. $B_{6}: 4.000 \mathrm{mg}$, vit. $B_{12}: 4.800 \mathrm{mg}$, ác. fólico: $1.200 \mathrm{mg}$, pantotenato de calcio: $12.000 \mathrm{mg}$, vit. C: $48.000 \mathrm{mg}$, biotina: $48 \mathrm{mg}$, colina: $65.000 \mathrm{mg}$, ácido nicotínico: $24.000 \mathrm{mg}$, Fe: $10.000 \mathrm{mg}, \mathrm{Cu}: 600 \mathrm{mg}, \mathrm{Mn}: 4.000 \mathrm{mg}, \mathrm{Zn}: 6.000 \mathrm{mg}$, I: $20 \mathrm{mg}$, Co: $2 \mathrm{mg}$, Se: $20 \mathrm{mg}$, 2) Vitamina C: sal cálcica 2-monofosfato de ácido ascórbico, $42 \%$ con principio activo, 3) Butil-Hidroxi-Tolueno, 4) Aglutinante, y 5) NRC (1993).

Table 1. Percentage composition of the reference diet. 1) Vitamin and mineral supplement (Supremais $\left.{ }^{\circledR}\right)$, composition per kilogram of product: vit. A: 1,200,000 UI, vit. $D_{3}: 200,000$ UI, vit. $E: 12,000 \mathrm{MG}$, vit. $K_{3}$ : 2,400 MG, vit. $B_{1}: 4,800$ MG, vit. $B_{2}: 4,800$ MG, vit. $B_{6}: 4,000$ MG, vit. $B_{12}: 4,800$ MG, folic acid: 1,200 mg, pantotenate of calcium: $12,000 \mathrm{mg}$, vit. $C: 48,000$ MG, biotine: $48 \mathrm{mg}$, coline: $65,000 \mathrm{mg}$, nicotinic acid: 24,000 mg, Fe: 10,000 mg, Cu: $600 \mathrm{mg}$, Mn: 4,000 mg, Zn: 6,000 mg, I: $20 \mathrm{mg}$, Co: 2 mg, Se: $20 \mathrm{mg}, 2$ ) Vitamin $\mathrm{C}$ : calcic salt 2-monofosfato of ascorbic acid, $42 \%$ with active principle, 3) Butil-Hidroxi-Toluen (antioxidant), 4) Agglutinant, and 5) NRC (1993).

\begin{tabular}{lc}
\hline Ingredientes & $\begin{array}{c}\text { Porcentaje } \\
(\%)\end{array}$ \\
\hline Maíz & 24,00 \\
Harina de soya & 31,18 \\
Harina de peces & 35,50 \\
Levadura deshidratada spray-dried & 4,00 \\
Fosfato bicálcico & 0,50 \\
Aceite de soya & 2,00 \\
Aceite de canola & 2,00 \\
Suplemento mineral y vitamínico (1) & 0,50 \\
Vitamina C (2) & 0,10 \\
Sal común & 0,10 \\
BHT (3) & 0,02 \\
Alginato (4) & 0,10 \\
\hline Total & 100,00 \\
Composición calculada (5) & \\
\hline Energía bruta (kcal·kg-1) & $4.343,00$ \\
Proteína bruta (\%) & 37,70 \\
Estrato etéreo (\%) & 7,37 \\
Fibra bruta (\%) & 2,98 \\
Calcio (\%) & 2,32 \\
Fósforo total (\%) & 1,26 \\
\hline
\end{tabular}


Tabla 2. Desarrollo de postlarvas de Macrobrachium rosenbergii en función de la sustitución del ensilado de maíz en la dieta. 1) Efecto lineal $(p<\mathbf{0 , 0 5})$; ganancia en peso $\left(Y=0,6128+\mathbf{0 , 0 0 2 5} \cdot \mathbf{X} ; \mathbf{R}^{2}=\mathbf{0 , 8 7}\right)$; biomasa final $(Y=25,7506$ $\left.+0,1399 \cdot X ; R^{2}=0,71\right) . C V$ : coeficiente de variación. *Diferencias con relación al tratamiento control por el test de Dunnett $(\mathbf{p}<\mathbf{0 , 0 5})$.

Table 2. Development of Macrobrachium rosenbergii postlarvae in function of the substitution of the corn silage in the diet. 1) Lineal effect $(p<0.05)$; gain of weight $\left(Y=0.6128+0.0025 \cdot X ; R^{2}=0.87\right)$; final biomass $(Y=25.7506$ $\left.+0.1399 \cdot X ; R^{2}=0.71\right) . C V$ : Variation coefficient. * Dunnett test diferences with relationship of the control treatment $(\mathbf{p}<0.05)$.

\begin{tabular}{lrrrrc}
\hline \multirow{2}{*}{ Variable } & \multicolumn{5}{c}{ Ensilado de maíz (\%) } \\
\cline { 2 - 6 } & \multicolumn{1}{c}{$\mathbf{0}$} & $\mathbf{8}$ & $\mathbf{1 6}$ & $\mathbf{2 4}$ & CV (\%) \\
\hline Peso inicial (g) & 0,12 & 0,12 & 0,12 & 0,12 & 20,14 \\
Peso final (g) & 0,74 & 0,74 & 0,78 & 0,79 & 5,40 \\
Aumento de peso (g) (1) & 0,62 & 0,62 & 0,66 & 0,67 & 6,42 \\
Consumo de ración (g·animal ${ }^{-1}$ ) & 37,31 & 40,60 & 38,71 & 37,50 & 8,04 \\
Biomasa final (g) (1) & 26,28 & 26,60 & 26,94 & 30,16 & 5,31 \\
Factor de conversión de la dieta & 1,78 & 1,87 & 1,75 & $1,48^{*}$ & 6,10 \\
Eficiencia proteica (1) & 1,59 & 1,49 & 1,60 & $1,90 *$ & 10,09 \\
Supervivencia & 88,75 & 90,00 & 88,75 & 95,00 & 6,35 \\
\hline
\end{tabular}
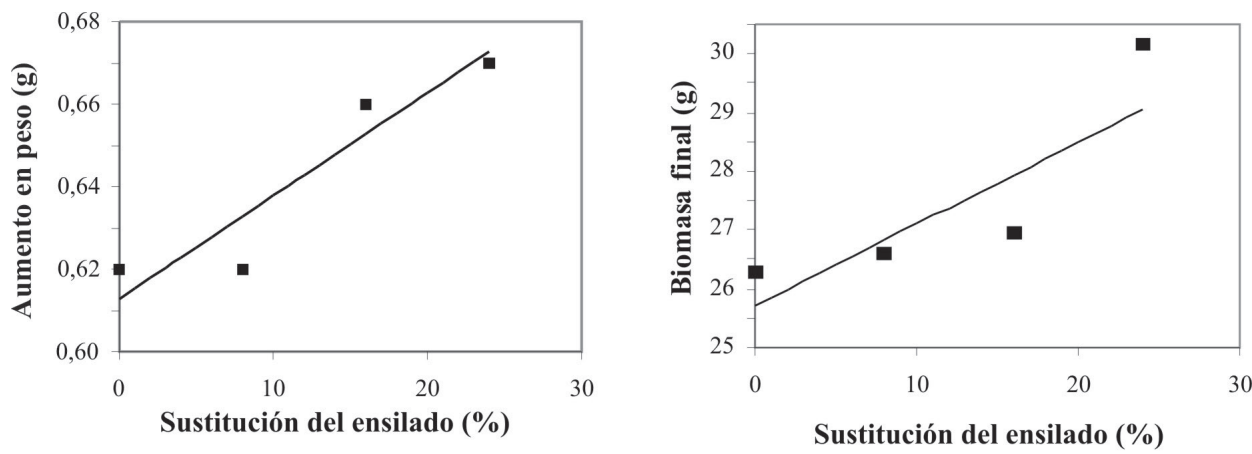

Figura 1. Aumento de peso y biomasa final de postlarvas de Macrobrachium rosenbergii en función de la sustitución del ensilado de maíz en la dieta.

Figure 1. Increase of weight and final biomass of Macrobrachium rosenbergii postlarvae in function of the substitution of the corn silage in the diet.

Según Biddle (1977), las necesidades energéticas de M. rosenbergii en las distintas etapas de su desarrollo son diferentes.

Valenti (1998) considera que los carbohidratos deben ser incluidos en las dietas comerciales para M. rosenbergii por una cuestión de economía, ya que además de ser una fuente económica de energía, contribuyen a la estabilidad de las mismas ya que actúan como aglutinantes. Tacon (1987) considera que los carbohidratos mejoran la palatabilidad y la estabilidad de las dietas. New (1995) recomienda un valor máximo de $40 \%$ de carbohidratos en las raciones para el camarón de agua dulce.

La obtención de estos antecedentes es de importancia para garantizar la formulación de dietas completas, palatables y de elevada digestibilidad, con el fin de obtener un buen rendimiento con el mínimo impacto sobre el medio ambiente. En virtud de las positivas proyecciones del crecimiento de la carcinicultura de agua dulce, enfatizado en una actividad sustentable económica y ambientalmente, que prioriza la maximización de la utilización y el no desperdicio de alimentos, se puede concluir que el ensilado de maíz puede sustituir totalmente el maíz en dietas para postlarvas de camarones de agua dulce de $M$. rosenbergii.

\section{AGRADECIMIENTOS}

Los autores agradecen a los profesores del Depar- 
tamento de Zootecnia de la Universidad Estatal de Maringá, al profesor Dr. Wagner Cotroni Valenti del Centro de Acuicultura de la Universidad Estatal Paulista, Jaboticabal-SP, a los amigos siempre presentes y en especial a nuestros familiares por su apoyo y dedicación.

\section{REFERENCIAS}

Bautista-Teruel, M.N., P.S. Eusébio \& T.P. Welsh. 2003. Utilization of peed pea, Pisum sativum, meal as a protein source in practical diets for juvenile tiger shrimp, Penaeus monodon. Aquaculture, 225: 121-131.

Biddle, G.N. 1977. The nutrition of freshwater prawns. En: J.A. Hanson \& H.L. Goodwin (eds.). Shrimp and prawns farming in the Western Hemisphere. Dowden, Hutchinson \& Ross, Stroudsbourg, pp. 272-291.

Bombeo-Tuburan, I., S. Fukumoto \& E.M. Rodríguez. 1995. Use of the golden apple snail, cassava, and maize as feed for the tiger shrimp, Penaeus monodon, in ponds. Aquaculture, 131: 91-100.

Cruz-Suarez, L.E., D. Ricque-Marie \& M. TapiaSalazar. 2001. Assessment of differently processed feed pea (Pisum sativum) meals and canola meal (Brassica sp.) in diets for blue shrimp (Litopenaeus stylirostris). Aquaculture, 196: 87-104.

Davis, D.A. \& C.R. Arnold. 1995. Effects of two extrusion processing conditions on the digestibility of four tiger prawn Penaeus vannamei. Aquaculture, 133: 287-294.

Euclydes, R.F. 1983. Manual de utilização do programa SAEG (Sistema para análises estatística e genética). Viçosa: UFV, 59 pp.

Jobim, C.C., U. Cecato \& M.W. do Canto. 2001. Utilização de silagem de grãos de cereais na alimentação animal. En: Simpósio sobre produção e utilização de forragens conservadas, 2001. Maringá, Paraná, Anais. Maringá: UEM/CCA/DZO, 1: 146-176.

Ling, S.W. 1969. The general biology and development of Macrobrachium rosenbergii. FAO Fish. Rep., 3(56): 589-606.

New, M.B. 1995. Status of freshwater prawn farming: a review. Aquaculture Res., Oxford, 26(1): 1-54.

New, M.B. 2000. History and global status of freshwater prawn farming. En: M.B. New \& W.C. Valenti (eds.). Freshwater prawn culture: the farming of Macrobrachium rosenbergii. Blackwell Science, Oxford, pp. 1-11.

Millamena, O.M. \& A.T. Triño. 1997. Low-cost feed for Penaeus monodon reared in tanks and under semi-intensive and intensive conditions in brackishwater ponds. Aquaculture, 154: 69-78.

Nacional Research Council (NRC). 1993. Nutrient requirements of warmwater fishes and shellfishes. National Academy Press, Washington, 102 pp.

Sudaryono, A., E. Tsvetnenko \& J. Hutabarat. 1999. Lupin ingredients in shrimp (Penaeus monodon) diets: influence of lupin species and types of meals. Aquaculture, 171: 121-133.

Tacon, A.G.J. 1987. The nutrition and feeding of farmed fish and shrimp: a training manual. FAO, GCP/RLA/075/ITA. FAO-FIELD Doc. 2 and 5, Brasilia, DF.

Valenti, V.C. 1998. Carcinicultura de água doce. Brasília: IBAMA, 383 pp.

Viana, M.T., M. Cervantes-Trujano \& R. SolanaSansores. 1994. Attraction and palatability activities in juvenile abalone (Haliotis fulgens): nine ingredients used in artificial diets. Aquaculture, 127: 19-28. 